\title{
Some clinically observed phenomena in extended contact lens wear
}

\author{
J. R. LARKE AND N. K. HIRJI \\ From the Department of Ophthalmic Optics, University of Aston, Birmingham
}

SUMMARY Two groups of volunteer subjects wearing extended-wear soft lenses were monitored over a period of 20 weeks. Of 6 factors measured only 1, corneal touch threshold, was found to show evidence of progressive change. However, a high incidence of lens surface deposits was encountered.

The ocular response to contact lens wear may be manifested in a number of ways. In addition to corneal thickness changes, previously reported by the authors (Hirji and Larke, 1979), the eye may show evidence of change in corneal topography, corneal sensitivity, refractive state, visual acuity, corneal epithelial integrity, and the appearance of the palpebral conjunctiva covering the tarsal area of the upper lid. In a controlled comparative study, now reported, the foregoing factors were examined in 3 groups of persons over a period of 20 weeks.

Fifty-seven volunteer Caucasian persons who came within a previously described acceptance profile were randomly divided into 3 groups. The first group (the non-solution group) were fitted with a high-water-content soft lens (Sauflon 85, Contact Lens Mfg.). The second group (the solution users) were fitted with similar lenses, which were removed and cleaned at 8-week intervals with a proprietary cleaning agent (Monoclens C40, Contact Lens Mfg.). In addition they instilled 1 drop of proprietary sterile saline on waking (Salette, Contact Lens Mfg.) and before retiring each day. The third group retained their spectacles and acted as controls. Data were collected from the subjects before lens wear and at 4-week intervals until a period of 20 weeks had elapsed.

\section{Results and discussion}

CORNEAL TOPOGRAPHY

Corneal curvature was measured with the aid of a Zeiss keratometer and supplementary topogometer. Measurements were taken at the central region of

Address for reprints: Dr J. R. Larke, Department of Ophthalmic optics, University of Aston, Gosta Green, Birmingham B4 7ET the cornea and at $15^{\circ}$ and $30^{\circ}$ apparent angular subtense in the horizontal and vertical meridians. The collected data were subjected to multifactorial and multiple regression analysis, which showed no statistically significant change $(P<0.001)$ in shape occurring in any of the monitored regions. Corneal shape change invariably accompanies the wearing of hard traditional contact lenses (Rengstorff, 1965). However, with the advent of Hydrogel lenses no statistically significant curvature changes have been reported.

The results of this study on corneal shape changes in periods of extended contact lens wear indicated a similar response to that reported on daily Hydrogel lens wear. The very differing physical properties of hard and Hydrogel lenses, coupled with the evidence of corneal shape change in the former but not in the latter, may support the hypothesis that corneal shape change in contact lens wear is at least partly mechanical in origin.

\section{CORNEAL TOUCH THRESHOLD LEVELS}

Corneal touch threshold was measured with the aid of a Cochet and Bonnet aesthesiometer mounted in a moveable $(X, Y)$ frame. Corneal touch threshold measurements were made in the vertical meridian, approximately $1 \mathrm{~mm}$ above the lower limbal margin of the cornea. A statistically significant change $(P<0.001)$ was observed in both groups of contact lens-wearing subjects. In addition a statistically significant difference $(P<0.001)$ was also observed between these 2 groups of subjects.

The collected data subjected to multiple regression analysis are graphically illustrated along with the regression equations in Fig. 1. It is apparent from Fig. 1 that the corneal touch threshold increased progressively throughout the 20 -week period of the study. At the end of about 12 weeks the reduction 


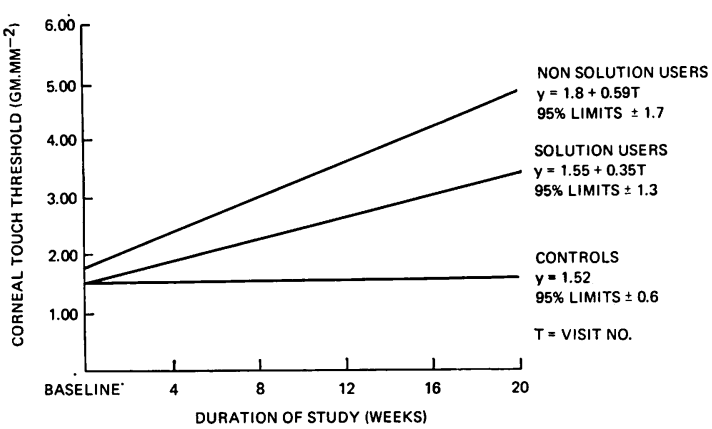

Fig. 1 Corneal touch threshold over 20 weeks of extended contact lens wear

in sensibility level is approximately equivalent to that which would have been achieved in about 1 day of traditional hard contact lens wear (Millodot, 1976). The difference between the 2 groups is not clearly understood at present. The lenses worn by both groups of participating subjects became covered with deposits as the study progressed. It is possible that the presence of these deposits contributed to the reduction in corneal sensibility. Among the group of patients who used proprietary solutions to clean the lenses the reduction in sensibility levels was less than among those who did not (though it should be borne in mind that the cleaning procedures allowed some corneal recovery time in this group of patients). However, this is only a tentative conclusion at present in view of the possibility that corneal touch threshold levels may also reflect subtle physiological changes occurring in the cornea.

\section{REFRACTIVE STATE}

Conventional refraction techniques were used to carry out over-refraction on the persons wearing contact lenses during the 20 weeks of the study. Multivariant regression analysis did not show a statistically significant change in this factor.

Although the ocular refraction without contact lenses in these groups of persons was not monitored in this study, in the absence of significant changes of over-refraction and corneal surface topography it is very unlikely that clinically significant changes in the ocular refraction over the duration of the study were in fact present and undetected. This strongly suggests a stable refractive pattern for this type of contact lens wear for the duration of the study.

\section{VISUAL ACUITY}

The visual acuity of the 3 groups of participating subjects was assessed on a conventional internally illuminated letter chart. The results showed no statistically significant evidence of change in the data over the study period nor between the 3 groups. However, empirical inspection of the data suggested some fluctuation, and, in particular, the standard deviation for the 2 groups of contact lens-wearing subjects appeared to be greater than that for the control subjects (Fig. 2). Such an interpretation was further supported by subjective comments by the patients of the appearance of 'watery' or somewhat unstable vision during the progress of the study. However, it is interesting to note that, although the contact lenses were subject to significant levels of deposits, this did not result in an associated depressed level of visual acuity for the group as a whole.

\section{EPITHELIAL INTEGRITY}

Epithelial integrity was assessed before and after contact lens wear with the aid of $1 \%$ sodium fluorescein and $2 \%$ rose Bengal, the eyes being examined by a slit lamp. An eyepiece graticule was used to assess the area of any staining observed during examination. It was found possible in almost all patients to find evidence of isolated punctate stain using either rose Bengal or fluorescein occupying less than $2 \%$ of the corneal surface on almost all the occasions on which the patients were examined. The writers regard this level of staining as a reflection of the 'normal' level of staining in patients at examining times. The incidence of vital staining involving more than $2 \%$ of the corneal surface was found not to differ $(\mathbf{P}<0.001)$ between the groups or between the periods of examination. Hence no evidence of epithelial trauma was detected in the present study.

APPEARANCE OF PALPEBRAL CONJUNCTIVA On completion of 20 weeks of extended contact lens wear the upper lids of all participating subjects were everted for an examination for the presence of giant conjunctival papillae. One instance of giant papillae in excess of $1 \mathrm{~mm}$ in diameter was encountered, while 5 instances of small raised (cobblestone) areas

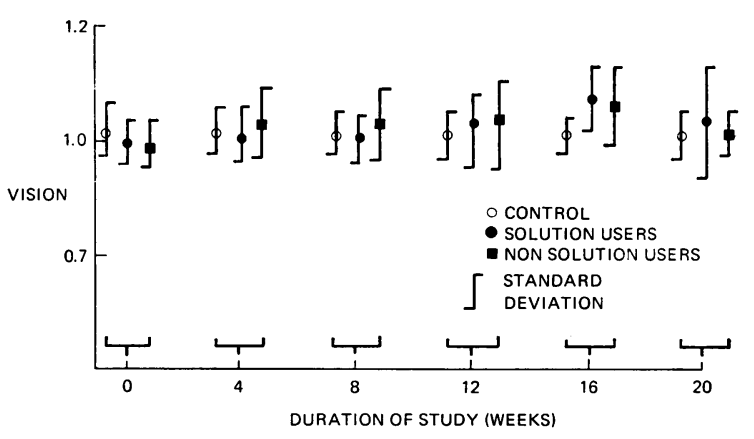

Fig. 2 Vision over 20 weeks of extended contact lens wear 
Table 1 Incidence and type of deposits

\begin{tabular}{|c|c|c|c|c|}
\hline \multicolumn{5}{|c|}{ Incidence $(\%)$ in week number } \\
\hline $\begin{array}{l}4 \\
29\end{array}$ & $\begin{array}{l}8 \\
39\end{array}$ & $\begin{array}{l}12 \\
50\end{array}$ & $\begin{array}{l}16 \\
63\end{array}$ & $\begin{array}{l}20 \\
55\end{array}$ \\
\hline Type & & $\%$ & & \\
\hline Crystalline & & $10 \cdot 8$ & & \\
\hline Film-like & & $7 \cdot 4$ & & \\
\hline Granular & & $12 \cdot 7$ & & \\
\hline Speckles & & $7 \cdot 4$ & & \\
\hline Others & & 8.9 & & \\
\hline
\end{tabular}

were noted. The appearance of giant conjunctival papillae is in all aspects similar to that first reported by Allensmith et al. (1977), and we have no further comments to add to those already reported in the literature.

\section{COMPLICATIONS}

In addition to the factors already disussed two further features arose in the study which we came to regard as complications of extended lens wear. The first was the high incidence of deposits on the lenses, and the second was a single instance of suspected growth of blood vessels into the cornea.

Deposits on soft contact lenses have been widely reported. In the present case the deposits had an apparently characteristic discrete appearance and were readily observed with the aid of a slit lamp by retroillumination. A record was kept of the incidence of deposits, and the results are summarised in Table 1. It is of interest to note that patients whose lenses were cleaned at 8-week intervals were still subject to a high level of deposits. It would seem wise for the efficacy and frequency of use of the cleaning compound used in this study to be closely examined.

During the course of the study the limbal arcades of one participating subject became congested. At the 12th week of wear an apparently unbranched and non-returning arcade was observed in the lower nasal quadrant of the left eye. The patient was advised to cease lens wear and was observed for a further 4 weeks. The eye became clear in less than 1 day, and no further problems were encountered. The subject was not allowed to resume lens wear.

\section{Conclusions}

The progressive increase in the corneal touch threshold of the contact lens-wearing subjects was the only factor monitored in this study to show evidence of progressive change. The loss in sensibility is approximately equivalent to what would be observed after 1 day's wear of hard contact lenses, though it is not possible to determine if the raised threshold is essentially mechanical or physiological in origin.

The very high incidence of lens surface deposits encountered among lens wearers is a serious complication to lens wear. The appearance of changes in the palpebral conjunctiva which Allensmith et al. (1977) has shown to arise from surface deposits strongly suggests that this problem requires to be resolved before extended lens wear becomes a practical proposition. Should a remedy to surface deposits be found, the nature of the ocular response to extended contact lens wear found in the present study suggests that such lenses are generally acceptable to the eye, at least in the short term.

\section{References}

Allensmith, M. R., Corb. D. R., Greiner, J. U., Henriquez, A. S., Simon, M. A., and Finnemore, U. M. (1977). Giant papillary conjunctivitis in contact lens wearers. American Journal of Ophthalmology, 83, 697-708.

Hirji, N. K., and Larke, J. R. (1979). Corneal thickness in extended contact lens wear. British Journal of Ophthalmology, 63, 274-276.

Millodot, M. (1976). Effect of the length of wear of contact lenses on corneal sensitivity. Acta Ophthalmologica, 54, 721-730.

Rengstorff, R. H. (1965). Corneal curvature and astigmatic changes subsequent to contact lens wear. Journal of the American Optometric Association. 36, 996-1000. 